\title{
The influence of lesion volume, perilesion resection volume, and completeness of resection on seizure outcome after resective epilepsy surgery for cortical dysplasia in children
}

\author{
Chima O. Oluigbo, MD, ${ }^{1}$ Jichuan Wang, PhD, ${ }^{3}$ Matthew T. Whitehead, MD, ${ }^{2}$ Suresh Magge, MD, ${ }^{1}$ \\ John S. Myseros, MD, ${ }^{1}$ Amanda Yaun, MD, ${ }^{1}$ Dewi Depositario-Cabacar, MD, ${ }^{4}$ \\ William D. Gaillard, MD, ${ }^{4}$ and Robert Keating, MD ${ }^{1}$
}

Departments of ${ }^{1}$ Neurosurgery, ${ }^{2}$ Radiology, ${ }^{3}$ Medical Statistics, and ${ }^{4}$ Neurology, Children's National Medical Center, Washington, DC

\begin{abstract}
OBJECT Focal cortical dysplasia (FCD) is one of the most common causes of intractable epilepsy leading to surgery in children. The predictors of seizure freedom after surgical management for FCD are still unclear. The objective of this study was to perform a volumetric analysis of factors shown on the preresection and postresection brain MRI scans of patients who had undergone resective epilepsy surgery for cortical dysplasia and to determine the influence of these factors on seizure outcome.
\end{abstract}

METHODS The authors reviewed the medical records and brain images of 43 consecutive patients with focal MRIdocumented abnormalities and a pathological diagnosis of FCD who had undergone surgical treatment for refractory epilepsy. Preoperative lesion volume and postoperative resection volume were calculated by manual segmentation using OsiriX PRO software.

RESULTS Forty-three patients underwent first-time surgery for resection of an FCD. The age range of these patients at the time of surgery ranged from 2 months to 21.8 years (mean age 7.3 years). The median duration of follow-up was 20 months. The mean age at onset was 31.6 months (range 1 day to 168 months). Complete resection of the area of an FCD, as adjudged from the postoperative brain MR images, was significantly associated with seizure control $(p=$ 0.0005). The odds of having good seizure control among those who underwent complete resection were about 6 times higher than those among the patients who did not undergo complete resection. Seizure control was not significantly associated with lesion volume $(p=0.46)$ or perilesion resection volume $(p=0.86)$.

CONCLUSIONS The completeness of FCD resection in children is a significant predictor of seizure freedom. Neither lesion volume nor the further resection of perilesional tissue is predictive of seizure freedom.

http://thejns.org/doi/abs/10.3171/2014.10.PEDS14282

KEY WORDS cortical dysplasia; children; lesion volume; seizure outcome; epilepsy

$\mathrm{F}$ OCAL cortical dysplasias (FCDs) are malformations of cortical development that result from abnormal neuronal migration and differentiation. They have been identified as one of the most common causes of intractable epilepsy leading to surgery in children. However, the predictors of freedom from seizures after surgical management for FCD are still being elucidated. Several clinical research groups have studied the impact of different radiological or clinicopathological factors on seizure outcome after extirpative surgery for FCD. ${ }^{-4,9,11-15,23,24,26,28,29}$

Recently, Rowland et al. ${ }^{22}$ reported on their meta-analysis of predictors of seizure freedom in the surgical management of FCD on the basis of results from 37 selected studies. They noted that complete resection of the anatomical or electrographic abnormality was the most important treatment-related factor predictive of seizure freedom. They also reported that temporal location, Palmini Type II histological classification, MRI detection, and presentation with partial seizures were each associated with a higher rate of postoperative seizure control.

There has never been, to our knowledge, a study focused on the volumetric analysis of radiological factors that can predict seizure outcome after surgery for FCD. Our objective in this study was to analyze volumetrically the factors in the preresection and postresection brain MRI scans of patients who had undergone resective epilepsy surgery

ABBREVIATIONS $F C D=$ focal cortical dysplasia; RV/LV = resection volume/lesion volume.

SUBMITTED June 4, 2014. ACCEPTED October 3, 2014.

INCLUDE WHEN CITING Published online March 13, 2015; DOI: 10.3171/2014.10.PEDS14282.

DISCLOSURE The authors report no conflict of interest concerning the materials or methods used in this study or the findings specified in this paper. 
for cortical dysplasia and the influence of these factors on seizure outcome. We examined the influence of preoperative lesion volume, resection volume, and the resection volume/lesion volume $(\mathrm{RV} / \mathrm{LV})$ ratio on seizure outcome. We also evaluated the impact of nonvolumetric radiological factors such as the completeness of resection, the location of the FCD and its proximity to eloquent cortex, and the relative impact of different clinicopathological factors in comparison with that of radiological factors on seizure outcome.

\section{Methods}

The medical records and high-resolution epilepsy protocol brain MRI scans of 43 consecutive patients who underwent surgical treatment for refractory epilepsy with focal MRI abnormalities and a pathological diagnosis of FCD were identified prospectively and reviewed retrospectively. This study was approved by the institutional review board of the Children's National Medical Center.

Each study was performed on either a $1.5-\mathrm{T}$ or a 3-T MRI scanner (GE Healthcare) and included at least the following pulse sequences: sagittal spoiled gradient-recalled echo T1-weighted images reformatted to show the axial and coronal planes, axial fast spin-echo T2-weighted images, axial diffusion-weighted images, axial T2*-weighted (star-weighted) angiograms, and coronal fat-saturated T2weighted images. Every preoperative examination included additional high-resolution coronal T2-weighted images through the hippocampus as part of our epilepsy brain MRI protocol. All of the examinations were of diagnostic quality. No exclusionary criteria prevented accurate evaluation.

A board-certified pediatric neuroradiologist reviewed the preoperative and postoperative brain MRI scans (obtained within 24-48 hours after surgery) from each patient to identify any signal alterations corresponding to the FCD and the completeness of resection on the respective preoperative and postoperative MR images. These pediatric neuroradiologists were not blinded.

Volumetric MRI analyses were performed using OsiriX PRO software, a Mac-based multimodality software for postprocessing and volume rendering. This software and its volumetric assessment methods have been demonstrated in numerous studies to have minimal bias and high intrarater and interrater reliabilities. ${ }^{16,30}$

We then performed manual segmentations of signal changes corresponding to the cortical dysplasia on the preoperative MR scans and the resection cavity on the postoperative MR scans. These data were used to calculate the preoperative lesion volumes and the postoperative resection volumes (Fig. 1).

We developed the RV/LV ratio as a surrogate volumetric measure. In this model, an $\mathrm{RV} / \mathrm{LV}$ ratio ratio of $>1 \mathrm{in}-$ dicates that the resection volume is greater than the lesion volume; therefore, if the postoperative scan demonstrated a gross-total lesion resection, then additional perilesional tissue had been resected by implication. In contradistinction, an RV/LV ratio ratio of $<1$ indicates incomplete lesion resection. However, there are inherent limitations to this model that are attributable to the dynamic changes

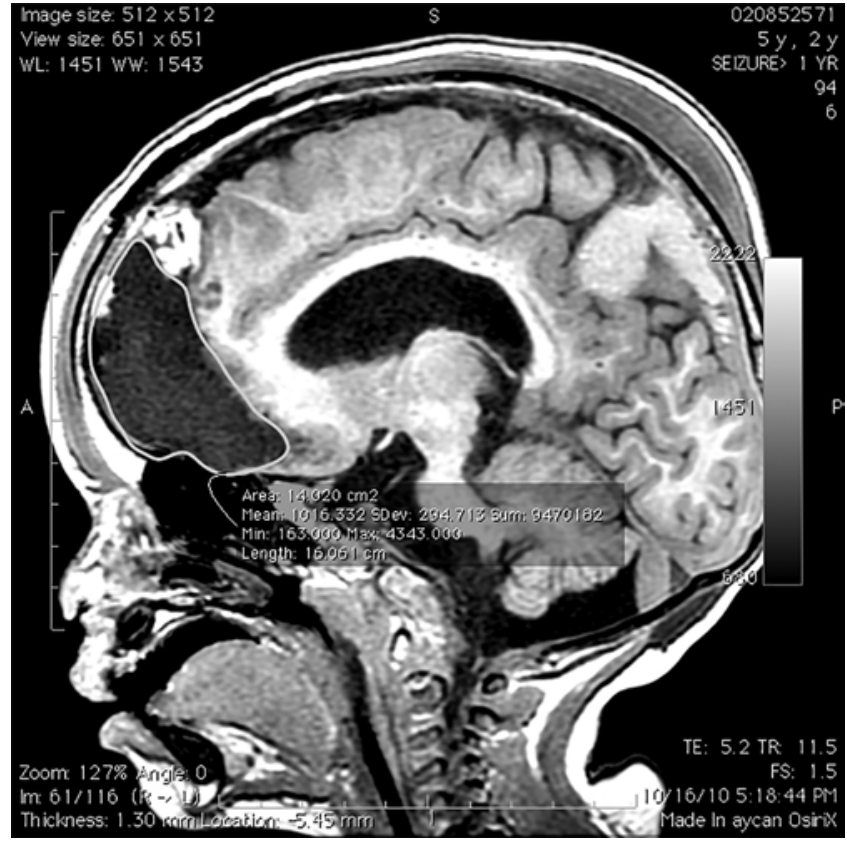

FIG. 1. Sagittal T1-weighted brain MR image showing the segmentation technique for delineating, in this case, the postsurgical resection cavity.

that occur in the operative bed. Collapse of the resection cavity can result in an underestimation of resection volume, a spuriously low RV/LV ratio, and a false-negative gross-total resection. Conversely, a large hematoma/seroma in the operative cavity can theoretically cause a falsely increased resection volume and RV/LV ratio. In practice, however, the resection cavity rarely increases in size; therefore, an $\mathrm{RV} / \mathrm{LV}$ ratio value of $>1$ reliably indicates complete lesion resection.

Our assessment of seizure outcomes was based on the Engel Epilepsy Surgery Outcome Scale, which was assessed at each patient's last clinic visit. We considered a patient to have a good outcome only if he or she was assessed to be in Engel Class I and was completely seizure free.

Other clinicopathologic and nonvolumetric radiological data acquired included duration of seizures, location of the FCD (temporal versus extratemporal), its adjacency to eloquent cortex, and the histopathological classification of the resected FCD.

The surgical approach entailed the use of neuronavigation in each case to confirm the position of the lesion (FCD) and to guide placement of the craniotomy. Electrocorticography was then performed before and after excision of the confirmed area of cortical dysplasia. Electrocorticography sometimes indicated areas of epileptiform activity adjacent to the margins of the area of a cortical dysplasia. When these perilesional areas were noneloquent cortical tissue, resection was extended to these areas. Thus, this "perilesional area" refers to the radially adjacent (noneloquent) cortex identified by electrocorticography as showing suspicious epileptiform activity. This situation can result in a resection volume that is larger than the lesion volume. Although some subcortical white matter is inadvertently resected, planned routine complete resection of white matter showing signal change 
("transmantle sign") all the way to the ventricle was not performed in these cases.

Univariate statistics were used to describe sample characteristics, and binary relationships were assessed by using the Student t-test and Fisher exact chi-square test. A p value of $<0.05$ was considered significant. A multivariate assessment of the impacts of different clinicopathological factors in comparison with those of the radiological factors on seizure control was then performed using a logistic regression model in which the dependent variable was the log odds of having good seizure control and the independent variables were complete resection, lesion volume, duration of seizures, temporal location, and histopathology results. Because the sample size was small, exact logistic regression was applied instead of regular logistic regression. A slope coefficient with a $p$ value of $<0.05$ was considered statistically significant.

\section{Results}

Of the 43 patients who underwent first-time surgery for resection of a cortical dysplasia, $65 \%$ were male. The mean age at onset was 31.6 months (range 1 day to 168 months), the mean age at surgery was 7.3 years (range 2 months to 21.8 years), and the median duration of followup was 20 months (Table 1). Complete sets of volumetric brain MRI scans of 41 patients were available for segmentation and volumetric analysis. Histopathological analysis was performed on all the lesions. Fifteen patients $(35 \%)$ had a Type 2B FCD. Twenty patients (47\%) had a Type 1 FCD, 6 patients (14\%) had a Type 2A FCD, and 2 patients $(5 \%)$ had an FCD of undetermined classification.

With binary statistical analysis, we found that complete resection of the area of the FCD, as judged from the postoperative brain MRI scan, was significantly associated with seizure control ( $p=0.0005$ ) (Table 2, Fig. 2).

Seizure control was not significantly associated with lesion volume. Although the mean lesion volume in patients with an Engel Class I outcome was lower than that in the rest of the patients $\left(12.2 \mathrm{vs} 16.4 \mathrm{~cm}^{3}\right)$, this difference was not significant ( $\mathrm{p}=0.46$ ) (Table 3, Fig. 3).

We then assessed whether seizure control was associated with increasing $\mathrm{RV} / \mathrm{LV}$ ratio values. We categorized each assessed patient as having an $\mathrm{RV} / \mathrm{LV}$ ratio of $\geq 1$ or $<$ 1. Among the patients with an Engel Class I outcome, most of them $(70.5 \%)$ had an $\mathrm{RV} / \mathrm{LV}$ ratio of $>1$. This result was reversed in the patients with a suboptimal seizure-control outcome, of whom the majority (approximately 58\%) had an $\mathrm{RV} / \mathrm{LV}$ ratio of $<1(\mathrm{p}=0.06)$ (Table 4 , Fig. 4$)$.

In redefining the parameters, we noted that a resection volume that exceeds the lesion volume $(\mathrm{RV} / \mathrm{LV}$ ratio $>1)$ becomes relevant only if the lesion itself is completely re-

TABLE 1. Descriptive statistics

\begin{tabular}{cc}
\hline \multicolumn{1}{c}{ Parameter } & Mean \pm SEM \\
\hline Age at surgery (yrs) & $7.3 \pm 0.8$ \\
\hline Duration of seizures (mos) & $56.2 \pm 7.9$ \\
\hline Age at seizure onset (mos) & $31.6 \pm 6.1$ \\
\hline Duration of follow-up (mos) & $26.1 \pm 3.5$ \\
\hline
\end{tabular}

TABLE 2. Complete resection and seizure control

\begin{tabular}{lccc}
\hline & \multicolumn{3}{c}{ No. of Patients } \\
\cline { 2 - 4 } \multicolumn{1}{c}{ Seizure Control } & $\begin{array}{c}\text { Complete } \\
\text { Resection }\end{array}$ & $\begin{array}{c}\text { Incomplete } \\
\text { Resection }\end{array}$ & Total \\
\hline Patients w/ Engel Class I & 16 & 2 & 18 \\
\hline $\begin{array}{l}\text { Patients w/ Engel Class II, } \\
\text { III, or IV }\end{array}$ & 9 & 16 & 25 \\
\hline Total & 25 & 18 & 43 \\
\hline
\end{tabular}

sected. This fact was considered in the context of previous data by Rowland et al. ${ }^{22}$ that indicated that complete lesion resection was associated significantly with good seizure control. Thus, a large resection volume when the lesion is not resected completely is not as significant as when the lesion is resected completely. Also, after complete resection of the area of a focal dysplasia, any further resection may cause untoward damage to normal perilesional tissue.

We therefore conducted a secondary analysis that encompassed patients with completely resected lesions, which was based on visual analysis. In this subset of patients, there was no difference in the mean \pm SEM RV/LV ratio of the patients with an Engel Class I outcome compared with that of the patients with an Engel Class II, III, or IV seizure-control outcome $(1.251 \pm 0.1892$ vs $1.308 \pm$ 0.2862 , respectively; $p=0.86)$. Thus, in patients with completely resected lesions, seizure control was not associated with increasing perilesional volumes (Table 5, Fig. 5).

Seizure control was not associated with the location of the lesion, histopathology results, or adjacency of the lesion to eloquent cortex (Fig. 6A-C).

Finally, a multivariate assessment of the relative impact of different clinicopathological factors in comparison with the radiological factors on seizure outcome (Table 6) indicated that complete resection has a positive effect on seizure control ( $p<0.035$, logistic regression) when controlling for covariates such as lesion volume, duration of seizures, temporal location, and histopathology results. The odds of having good seizure control among those who underwent complete resection were 6.1 times $(95 \%$

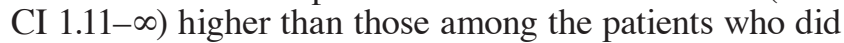
not undergo complete resection. In the logistic regression analysis, no predictors other than complete resection had a significant effect on seizure control.

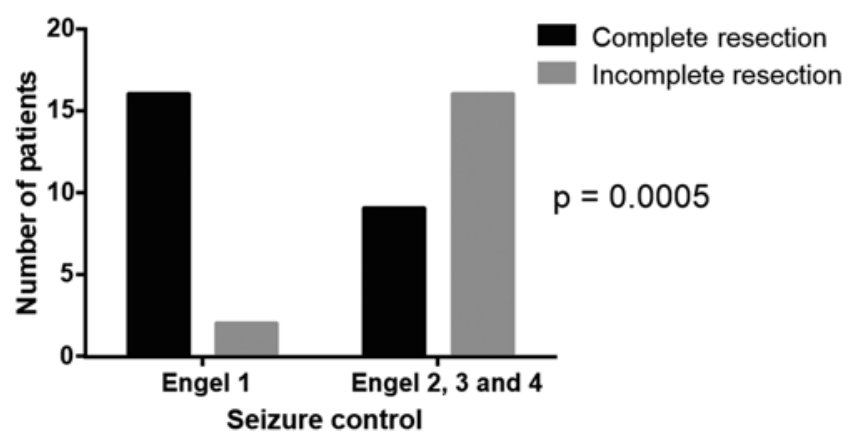

FIG. 2. Complete resection is significantly associated with seizure control. 
TABLE 3. Relationship of lesion volume to seizure control

\begin{tabular}{lc}
\hline \multicolumn{1}{c}{ Seizure Control } & Lesion Vol $\left(\mathrm{cm}^{3}\right)^{\star}$ \\
\hline Patients w/ Engel Class I $(\mathrm{n}=17)$ & $12.24 \pm 2.264$ \\
\hline Patients w/ Engel Class II, III, or IV $(\mathrm{n}=24)$ & $16.43 \pm 4.475$ \\
\hline
\end{tabular}

* Presented as the mean \pm SEM.

\section{Discussion}

We found that in our cohort of 43 patients who had undergone first-time surgery for the resection of an FCD, complete resection of the radiological abnormality was a significant predictor of seizure freedom. The odds of having good seizure control among those who underwent complete resection were approximately 6 times higher than among the patients who did not undergo complete resection. Lesion volume was not a predictor of seizure freedom, and after complete resection of the radiological abnormality, the further resection of perilesional tissue did not seem to improve seizure outcome.

The prevalence of FCDs in children being evaluated for intractable epilepsy is estimated to range between $5 \%$ and $25 \%$ depending on the imaging technique used to establish the diagnosis. ${ }^{2}$ Therefore, it is not surprising that they are one of the most common indications for epilepsy surgery in children with intractable focal epilepsy. These malformations of neuronal migration were not recognized as an entity until they were described by Taylor et al. in $1971 .^{25}$ The development of MRI in the 1980s boosted the recognition of these lesions in patients who had been described previously as having "cryptogenic" focal epilepsy. With the evolution in MRI techniques and technology and the adoption of detailed epilepsy imaging protocols using 3-T magnets, smaller lesions began to be identified.

The identification of FCDs is important. Multiple studies and meta-analyses have shown that the identification of a structural epileptogenic substrate in patients with intractable focal epilepsy is associated with outcomes that are superior to those in patients in whom no structural lesion is identified. $1,4,8,9,11,12,14,22-24,26,29$

There have also been attempts to predict seizurecontrol outcome in these patients on the basis of their radiological appearance and multiple clinicopathological

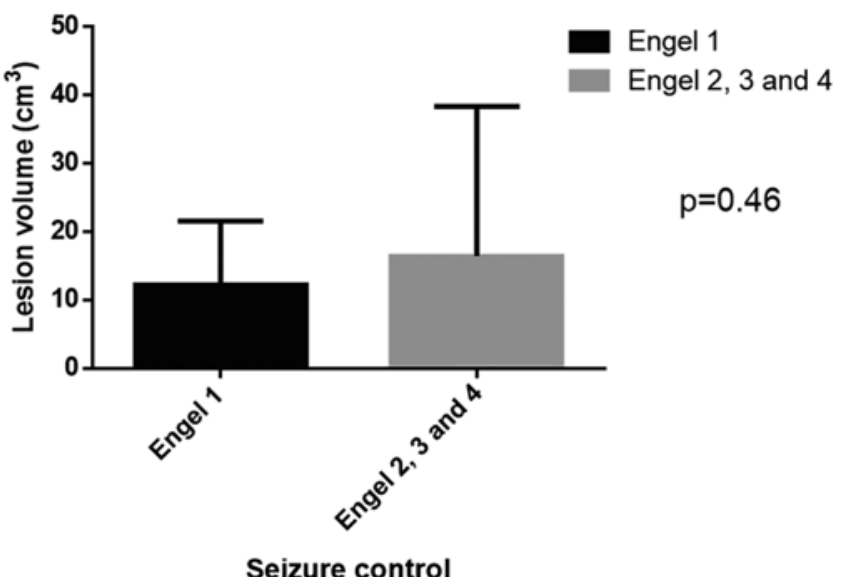

FIG. 3. Seizure control is not significantly associated with lesion volume.
TABLE 4. Relationship of RV/LV to seizure control

\begin{tabular}{lccc}
\hline & \multicolumn{3}{c}{ No. of Patients } \\
\cline { 2 - 4 } Seizure Control & RV/LV $\geq 1$ & RV/LV <1 & Total \\
\hline Patients w/ Engel Class I & 12 & 5 & 17 \\
\hline $\begin{array}{l}\text { Patients w/ Engel Class II, III, } \\
\text { or IV }\end{array}$ & 10 & 14 & 24 \\
\hline Total & 22 & 19 & 41 \\
\hline
\end{tabular}

features. Wang et al. ${ }^{28}$ reported on the transmantle sign noted on brain MR images as a predictor of good outcome after the resection of these lesions. The location of these lesions (temporal versus extratemporal) has also been investigated as a predictive factor for postoperative seizure control. ${ }^{22}$ Adjacency of these lesions to eloquent cortex, which may limit a surgeon's aggressiveness in attempting to resect them, has also been identified as a predictive factor, because the residual lesion is associated with poor seizure control. ${ }^{11}$

The histopathological classification of these lesions has also been linked to surgical outcome. ${ }^{22}$ In 2004, Palmini et al. ${ }^{20}$ classified these lesions on a histopathological basis as Type 1 or 2 FCD. Type 1 FCD is characterized by dyslamination or disruption of the normal cortical architecture with no dysmorphic or balloon cells seen. In Type 2 FCD (also referred to as the Taylor-type FCD), in addition to cortical dyslamination, dysmorphic cells (Type 2A) or characteristic balloon cells (Type 2B) are found. In 2011, an ad hoc task force of the International League Against Epilepsy proposed a consensus classification on the clinicopathologic spectrum of FCDs, and a Type 3 classification, in which cortical dyslamination is associated with another potentially epileptogenic lesion in the same brain area or lobe, was included. ${ }^{3}$ Type $2 \mathrm{~B}$ lesions have been associated with improved seizure outcome after their resection. ${ }^{22}$

The extent of resection has been linked definitively to improved seizure-control outcome. In a meta-analysis of 37 selected studies, Rowland et al. ${ }^{22}$ identified complete resection of the radiological or electrographic abnormality as the most important treatment-related factor that is predictive of a good seizure-control outcome. Our study was focused on volumetric quantification on the relative influence of lesion volume and resection volume on seizure outcome after surgery for an FCD.

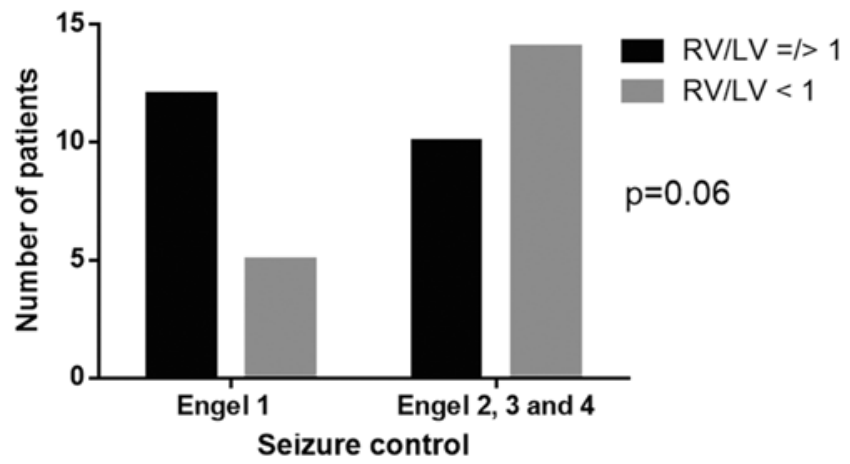

FIG. 4. Relationship of seizure control to increasing RV/LV ratio. 
TABLE 5. Influence of further perilesional resection volume on seizure control in 23 patients with completely resected lesions versus seizure outcome

\begin{tabular}{lc}
\hline \multicolumn{1}{c}{ Seizure Control } & RV/LV Ratio* \\
\hline Patients w/ Engel Class I $(n=15)$ & $1.251 \pm 0.1892$ \\
\hline Patients w/ Engel Class II, III, or IV $(n=8)$ & $1.308 \pm 0.2862$ \\
\hline * Presented as the mean \pm SEM. &
\end{tabular}

The value of resecting tissue surrounding the identified area of a cortical dysplasia is unclear. Wagner et al..$^{26}$ noted that in FCD Type 2B, resection of the subcortical radiological abnormality was not necessary for seizure freedom, provided the cortical abnormality had been completely resected. In our study, we noted that when considering only patients with completely resected cortical lesions, seizure control was not significantly associated with increasing perilesional volumes.

\section{Volumetrics and Epileptogenesis}

Such considerations of the perilesional volume of resection may have implications for understanding the underlying basis of epileptogenesis in FCDs, which remains unclear. Proposed mechanisms include the presence of abnormal cells, such as balloon cells, in focal malformations of cortical development, which act as pacemakers for epileptiform discharges. There is some evidence to suggest that balloon cells display intrinsic hyperexcitability, possibly because of a modification of $N$-methyl-D-aspartate receptors or decreased sensitivity of the $\gamma$-aminobutyric acid A receptors in these pacemaker cells..$^{10,18,19,31,32}$ In more diffuse cortical dysplasia, there may be abnormal connectivity of the cellular aggregate, which is associated with reorganization of cortical circuitry, and axons that would normally project out of the epileptic zone may also be interrupted and instead make excitatory synapses locally. These recurrent excitatory connections may cause feed-forward connectivity loops that drive the excitatory and consequent epileptic process.

In this context, volumetric considerations such as the $\mathrm{RV} / \mathrm{LV}$ ratio, which is a surrogate measure of perilesion

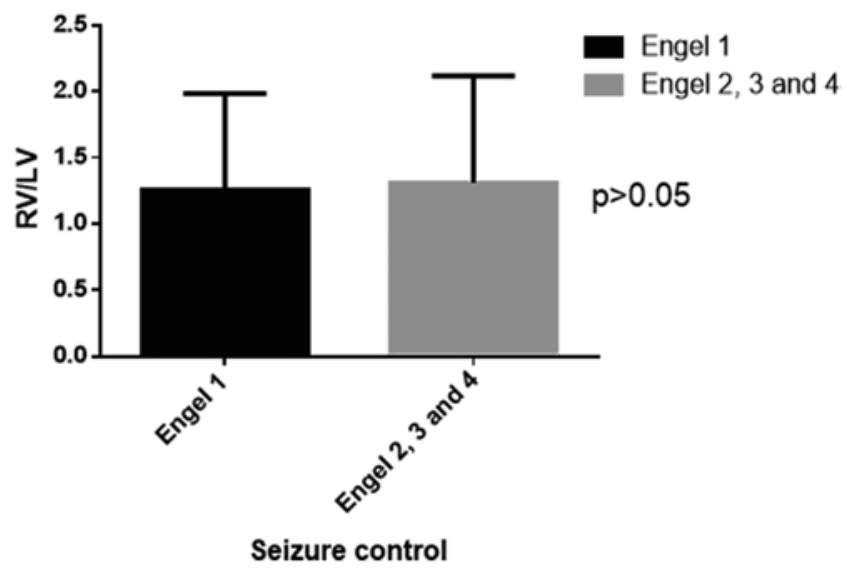

FIG. 5. Completely resected lesions: the influence of further perilesion resection volume. resection in patients with completely resected cortical lesions, may represent an estimation of the extent of disruption of these extralesional epilepsy networks.

Our finding that increasing RV/LV ratio values were inferior to the completeness of lesion resection in determining seizure freedom may point to the fact that intralesional networks are dominant over extralesional networks in determining epileptogenesis in cortical dysplasias. However, there is no direct evidence in the literature to suggest that lesion volume has any direct effect on outcome.

The general tendency among those who perform epilepsy surgery is to resect subcortical tissue after the complete resection of the cortical abnormality, which is probably because there is sometimes an associated subcortical radiological abnormality such as the transmantle sign. The

Seizure control and location

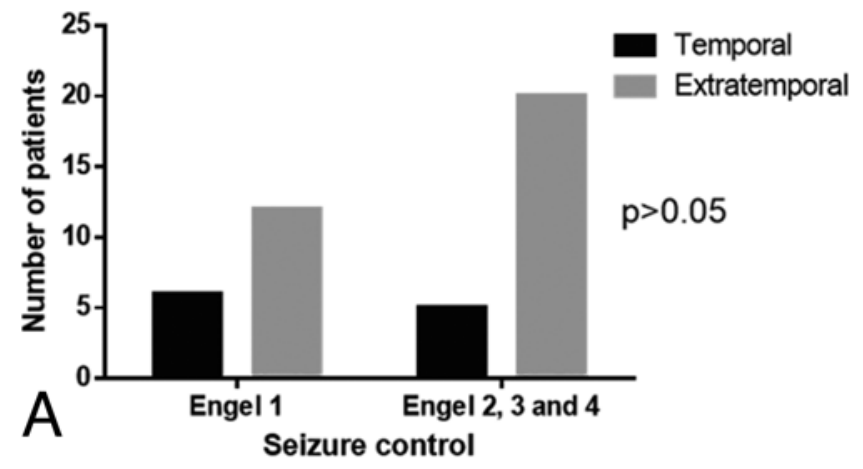

Seizure control and Histopathology

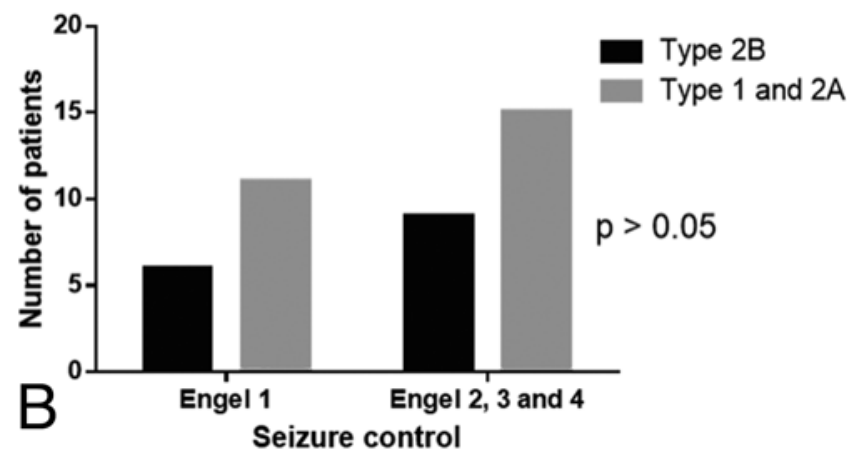

Seizure control and adjacent eloquent cortex

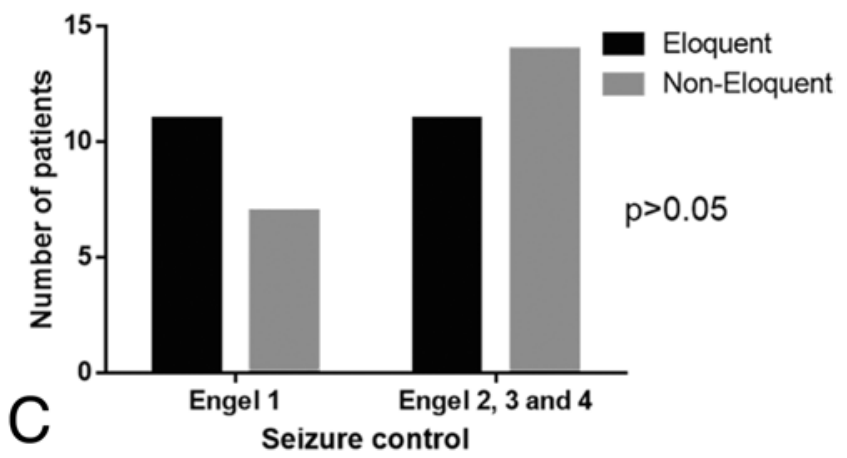

FIG. 6. Relationships of location of the lesion (A), histopathology results (B), and adjacency to eloquent cortex (C) to seizure control. 
TABLE 6. Exact logistic regression results

\begin{tabular}{lccc}
\hline \multicolumn{1}{c}{ Parameter } & $\begin{array}{c}\text { Exact OR } \\
\text { Estimate }\end{array}$ & \multicolumn{1}{c}{$95 \% \mathrm{Cl}$} & p Value \\
\hline Complete resection & 6.062 & $1.108-\infty$ & 0.0350 \\
\hline Lesion volume & 1.004 & $0.964-1.043$ & 0.8735 \\
\hline Duration & 1.001 & $0.987-1.016$ & 0.8643 \\
\hline Temporal & 0.607 & $0.060-8.388$ & 0.9000 \\
\hline Histopathological results & 1.261 & $0.195-15.467$ & 1.000 \\
\hline
\end{tabular}

surgeon, therefore, aims to resect this subcortical lesion, sometimes as deep as the underlying ventricles. Wagner et al. ${ }^{26}$ as described above, already found that such subcortical resection does not correlate with improved seizure control.

Although these findings may suggest that perilesional (extralesional) epilepsy networks are not the predominant factor in the epileptogenesis of cortical dysplasias, it may be more precise to state that subcortical (perpendicularly oriented) epilepsy networks are not critical in determining epileptogenicity. Therefore, there remains a possibility that radially oriented extralesional networks may still be relevant in the epileptogenicity of these lesions. It is unlikely, however, that the contribution of radially oriented network connections can be adequately assessed because of the tendency to limit the lateral resection beyond the anatomical limits of these lesions as a result of concerns for incurring neurological deficits, especially when they are located adjacent to eloquent cortex.

The findings on intraoperative electrocorticography of electrographic abnormality on cortex situated radially beyond the structural limits of the radiographically identified lesion may support the existence of such radially oriented networks.

In our study, adjacency to eloquent cortex was not associated with poor seizure-control outcome. It is, however, reasonable to expect that adjacency to eloquent cortex may limit a surgeon's ability to achieve a complete extirpation of the lesion. The inability of the statistical analysis to detect an influence from proximity to eloquent cortex may be related to the fact that our small cohort of patients was not sufficiently statistically powered to identify a difference.

Similarly, complete lesion resection and its highly significant relationship to seizure freedom are likely related to the complete disruption of the intralesional epilepsy networks and the hyperactive pacemaker cells contained within the lesion. This likely explains why completeness of resection supercedes the volume of the lesion in determining seizure freedom after surgery for cortical dysplasias.

The confirmation in our study and other studies of the critical importance of complete resection of the cortical abnormality in determining seizure freedom after surgery for the resection of a cortical dysplasia raises the need for additional studies into techniques to identify and delineate the areas of FCDs, especially in the pathological types, which are not usually evident on MRI. Methods for the automated detection of areas of FCDs on imaging using automated segmentation and postacquisition image-processing algorithms aimed at detecting cortical thickness or gray matter-white matter junction blurring are being developed and, in some situations, are superior to expert human visual analysis. ${ }^{27}$

The influence of the 3-T versus the 1.5-T scanner on the detection of the lesion or the resection to lesion volume ratios were not specifically analyzed in our study. However, other studies have shown that the use of 3-T MRI in patients suspected of having a Type 2 FCD improves the detection rate and the lesion characterization because of the transmantle sign being more clearly visible at $3 \mathrm{~T} \cdot{ }^{17}$

Other novel techniques include the use of optical coherence tomography and the use of high-frequency ultrasonography for micrometer-level intraoperative resolution and the delineation of these lesions. ${ }^{21}$

On a more practical level, some centers have used MRI for intraoperative confirmation of complete lesion resection, which may be especially important in patients with lesions located in regions with complex 3D gyral anatomy, such as insular lesions. Once again, the limits of MRI for these lesions will still be manifest here. For example, a subtle FCD detected on thin-section images from an epilepsy protocol brain MRI study may be difficult to see on intraoperative MR images because the expected operative intracranial blood products and gas can distort the magnetic field, alter the local signal, and obscure potential residual lesions. These difficulties underscore the need for improved intraoperative MR techniques for the delineation of residual lesions.

The limitations of our study are related to the relatively small size of the cohort and the nonblinded assessments of the extents of resection and lesion volumes. Our assessments of the completeness of resection depended on visual evaluation by a pediatric neuroradiologist. The assessments of lesion volumes and resection volumes were based on MRI volumetric analysis performed using the OsiriX PRO software, a Mac-based multimodality software for postprocessing and volume rendering. However, this software and its volumetric assessment methods have been shown in numerous studies to have minimal bias and high intrarater and interrater reliabilities..$^{16,30}$

\section{Conclusions}

In our cohort of 43 pediatric patients who underwent first-time surgery for the resection of an FCD, complete resection of the radiological abnormality was a significant predictor of seizure freedom. The odds of having good seizure control among those who underwent complete resection was about 6 times higher than those in patients who did not undergo complete resection. Lesion volume was not a predictor of seizure freedom. Finally, after complete resection of the radiological abnormality, further resection of perilesional tissue did not seem to improve seizure outcome.

\section{References}

1. Alexandre V Jr, Walz R, Bianchin MM, Velasco TR, TerraBustamante VC, Wichert-Ana L, et al: Seizure outcome after surgery for epilepsy due to focal cortical dysplastic lesions. Seizure 15:420-427, 2006

2. Bast T, Ramantani G, Seitz A, Rating D: Focal cortical dysplasia: prevalence, clinical presentation and epilepsy in children and adults. Acta Neurol Scand 113:72-81, 2006 
3. Blümcke I, Thom M, Aronica E, Armstrong DD, Vinters HV, Palmini A, et al: The clinicopathologic spectrum of focal cortical dysplasias: a consensus classification proposed by an ad hoc Task Force of the ILAE Diagnostic Methods Commission. Epilepsia 52:158-174, 2011

4. Chang EF, Wang DD, Barkovich AJ, Tihan T, Auguste KI, Sullivan JE, et al: Predictors of seizure freedom after surgery for malformations of cortical development. Ann Neurol 70:151-162, 2011

5. Chassoux F, Landré E, Mellerio C, Turak B, Mann MW, Daumas-Duport C, et al: Type II focal cortical dysplasia: electroclinical phenotype and surgical outcome related to imaging. Epilepsia 53:349-358, 2012

6. Chern JJ, Patel AJ, Jea A, Curry DJ, Comair YG: Surgical outcome for focal cortical dysplasia: an analysis of recent surgical series. J Neurosurg Pediatr 6:452-458, 2010

7. Cohen-Gadol AA, Ozduman K, Bronen RA, Kim JH, Spencer DD: Long-term outcome after epilepsy surgery for focal cortical dysplasia. J Neurosurg 101:55-65, 2004

8. Fauser S, Bast T, Altenmüller DM, Schulte-Mönting J, Strobl K, Steinhoff BJ, et al: Factors influencing surgical outcome in patients with focal cortical dysplasia. J Neurol Neurosurg Psychiatry 79:103-105, 2008

9. Fauser S, Schulze-Bonhage A, Honegger J, Carmona H, Huppertz HJ, Pantazis G, et al: Focal cortical dysplasias: surgical outcome in 67 patients in relation to histological subtypes and dual pathology. Brain 127:2406-2418, 2004

10. Hablitz JJ, DeFazio RA: Altered receptor subunit expression in rat neocortical malformations. Epilepsia 41 (Suppl 6):S82-S85, 2000

11. Hader WJ, Mackay M, Otsubo H, Chitoku S, Weiss S, Becker $\mathrm{L}$, et al: Cortical dysplastic lesions in children with intractable epilepsy: role of complete resection. J Neurosurg 100 (2 Suppl Pediatrics):110-117, 2004

12. Kim DW, Lee SK, Chu K, Park KI, Lee SY, Lee CH, et al: Predictors of surgical outcome and pathologic considerations in focal cortical dysplasia. Neurology 72:211-216, 2009

13. Kral T, Clusmann H, Blümcke I, Fimmers R, Ostertun B, Kurthen M, et al: Outcome of epilepsy surgery in focal cortical dysplasia. J Neurol Neurosurg Psychiatry 74:183-188, 2003

14. Krsek P, Maton B, Jayakar P, Dean P, Korman B, Rey G, et al: Incomplete resection of focal cortical dysplasia is the main predictor of poor postsurgical outcome. Neurology 72:217-223, 2009

15. Krsek P, Pieper T, Karlmeier A, Hildebrandt M, Kolodziejczyk D, Winkler P, et al: Different presurgical characteristics and seizure outcomes in children with focal cortical dysplasia type I or II. Epilepsia 50:125-137, 2009

16. Li D, Hodge J, Wei XC, Kirton A: Reduced ipsilesional cortical volumes in fetal periventricular venous infarction. Stroke 43:1404-1407, 2012

17. Mellerio C, Labeyrie MA, Chassoux F, Roca P, Alami O, Plat M, et al: 3T MRI improves the detection of transmantle sign in type 2 focal cortical dysplasia. Epilepsia 55:117-122, 2014

18. Möddel G, Jacobson B, Ying Z, Janigro D, Bingaman W, González-Martínez J, et al: The NMDA receptor NR2B subunit contributes to epileptogenesis in human cortical dysplasia. Brain Res 1046: 10-23, 2005

19. Najm IM, Ying Z, Babb T, Mohamed A, Hadam J, LaPresto E, et al: Epileptogenicity correlated with increased N-methylD-aspartate receptor subunit NR2A/B in human focal cortical dysplasia. Epilepsia 41:971-976, 2000

20. Palmini A, Najm I, Avanzini G, Babb T, Guerrini R, FoldvarySchaefer N, et al: Terminology and classification of the cortical dysplasias. Neurology 62 (6 Suppl 3):S2-S8, 2004

21. Roper SN, Moores MD, Gelikonov GV, Feldchtein FI, Beach NM, King MA, et al: In vivo detection of experimentally induced cortical dysgenesis in the adult rat neocortex using optical coherence tomography. J Neurosci Methods 80:9198, 1998

22. Rowland NC, Englot DJ, Cage TA, Sughrue ME, Barbaro NM, Chang EF: A meta-analysis of predictors of seizure freedom in the surgical management of focal cortical dysplasia. J Neurosurg 116:1035-1041, 2012

23. Sarkis RA, Jehi LE, Bingaman WE, Najm IM: Surgical outcome following resection of rolandic focal cortical dysplasia. Epilepsy Res 90:240-247, 2010

24. Tassi L, Garbelli R, Colombo N, Bramerio M, Lo Russo G, Deleo F, et al: Type I focal cortical dysplasia: surgical outcome is related to histopathology. Epileptic Disord 12:181-191, 2010

25. Taylor DC, Falconer MA, Bruton CJ, Corsellis JA: Focal dysplasia of the cerebral cortex in epilepsy. J Neurol Neurosurg Psychiatry 34:369-387, 1971

26. Wagner J, Urbach H, Niehusmann P, von Lehe M, Elger CE, Wellmer J: Focal cortical dysplasia type IIb: completeness of cortical, not subcortical, resection is necessary for seizure freedom. Epilepsia 52:1418-1424, 2011

27. Wagner J, Weber B, Urbach H, Elger CE, Huppertz HJ: Morphometric MRI analysis improves detection of focal cortical dysplasia type II. Brain 134:2844-2854, 2011

28. Wang DD, Deans AE, Barkovich AJ, Tihan T, Barbaro NM, Garcia PA, et al: Transmantle sign in focal cortical dysplasia: a unique radiological entity with excellent prognosis for seizure control. J Neurosurg 118:337-344, 2013

29. Widjaja E, Otsubo H, Raybaud C, Ochi A, Chan D, Rutka JT, et al: Characteristics of MEG and MRI between Taylor's focal cortical dysplasia (type II) and other cortical dysplasia: surgical outcome after complete resection of MEG spike source and MR lesion in pediatric cortical dysplasia. Epilepsy Res 82:147-155, 2008

30. Win Mar Salmah J, Noorfizura A, Mohd Shafie A, Helmy A, Salmi AR, Naing L: Hippocampal MR volumetric studies in paediatric patients with epilepsy and normal controls. Neuroradiol J 24:503-510, 2011

31. Ying Z, Babb TL, Comair YG, Bingaman W, Bushey M, Touhalisky K: Induced expression of NMDAR2 proteins and differential expression of NMDAR1 splice variants in dysplastic neurons of human epileptic neocortex. J Neuropathol Exp Neurol 57:47-62, 1998

32. Ying Z, Babb TL, Mikuni N, Najm I, Drazba J, Bingaman W: Selective coexpression of NMDAR2A/B and NMDAR1 subunit proteins in dysplastic neurons of human epileptic cortex. Exp Neurol 159:409-418, 1999

\section{Author Contributions}

Conception and design: Oluigbo, Gaillard. Acquisition of data: Oluigbo. Analysis and interpretation of data: Oluigbo, Whitehead. Drafting the article: Oluigbo. Critically revising the article: Oluigbo, Whitehead, Magge, Myseros, Depositario-Cabacar, Gaillard, Keating. Reviewed submitted version of manuscript: Oluigbo, Magge, Myseros, Yaun, Depositario-Cabacar, Gaillard, Keating. Approved the final version of the manuscript on behalf of all authors: Oluigbo. Statistical analysis: Oluigbo, Wang. Study supervision: Oluigbo, Gaillard, Keating.

\section{Supplemental Information}

\section{Previous Presentation}

Portions of this work were presented at the 37th Annual Meeting of the American Society of Pediatric Neurosurgeons, Guanacaste, Costa Rica, January 26-31, 2014.

\section{Correspondence}

Chima O. Oluigbo, Department of Neurosurgery, Children's National Medical Center, 111 Michigan Ave. NW, Washington, DC 20010. email: coluigbo@cnmc.org. 